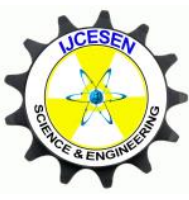

Copyright (C) IJCESEN
International Journal of Computational and

Experimental Science and $\boldsymbol{E N g i n e e r i n g}$

(IJCESEN)

Vol. 6-No.3 (2020) pp. 169-172

http://dergipark.org.tr/en/pub/ijcesen

Research Article

\title{
Calculated hyperfine coupling constants of some sigma-type radicals
}

\author{
Fatih UCUN*, Sadun KÜÇÜK \\ Süleyman Demirel University, Science and Arts Faculty, Physics Department, 32200, Isparta-Turkey \\ * Corresponding Author : fatihucun@sdu.edu.tr \\ ORCID: 0000-0001-7464-7788
}

\section{$\underline{\text { Article Info: }}$}

DOI: $10.22399 /$ ijcesen.750537

Received : 10 June 2020

Accepted : 11 November 2020

\section{Keywords}

Hyperfine Constant

EPR

Radical

Density Functional Theory

Simulation

\begin{abstract}
$\underline{\text { Abstract: }}$
The hyperfine coupling constants of some $\sigma$-type radicals were calculated by using the DFT(B3LYP) method with LanL2DZ basic set. These are phenyl, naphthyl, pyridyl, and quinolyl radicals. Also, the study was enriched by the calculations of the spin densities for all the radicals. From the results it was concluded that the spin density of the unpaired electron is mainly in one sp-hybrid orbital of carbon atom from which hydrogen is removed. The electron paramagnetic resonance (EPR) spectra of all the radicals are generally based on the hyperfine splitting belonging to only the close hydrogen or nitrogen atoms of the benzene ring to the unpaired electron since the effect of the other atoms is disappeared in the linewith of the EPR spectrum lines. The EPR spectra were also simulated by using their calculated isotropic hyperfine coupling constants and, compared with the experimental data. It was found a good agreement between them.
\end{abstract}

\section{Introduction}

Analysis of electron paramagnetic resonance (EPR) spectra of $\sigma$-type radicals give detailed information about the distributions of the spin density of the unpaired electron. The bigger hyperfine coupling constant means the higher spin density. Pyridyl (2-, 3-, and 4-) [1], 2-, 3-, and 4-quinolyl and 4isoquinolyl [2] radicals generated in argon matrices after UV irradiation were studied by EPR spectroscopy. All these radicals were found to be $\sigma$ type radicals. The EPR spectra of the radicals were readily resolved by the identifications of the hyperfine values of the protons of the radicals. The results indicated a little effect of the fused benzene ring upon the nature of the semi filled orbitals of the radicals. Some authors have also reported the observation of the carbon-13 spectra of phenyl, 2pyridyl, and 2-pyrimidinyl radicals [3]. A specific chemical reaction was used in the preparation of these radicals, and the radicals were trapped in an inert matrix at $77^{\circ} \mathrm{K}$ using a rotating cryostat. They found that the unpaired electron is localized mainly in one sp-hybrid orbital of the carbon atom form which proton is removed and, is not delocalized in the orbital system of the aromatic ring. The electronic ground states of phenyl, 1- and 2naphthyl, 1- and 9-anthracyl, and 1-pyrenyl radicals were determined by means of EPR in order to find if a crossing of $s$ and $p$ levels occurs as the aromatic ring system is expanded [4]. In all cases the unpaired electron was found to occupy on the essentially nonbonding orbital corresponding to the broken bond.

Some authors have showed that the density functional theory (DFT) method could be helpful to make proper assignments of complex EPR spectra and, to determine the main features of the distributions of the spin densities of overcrowded polycyclic aromatic hydrocarbon radical cations [5]. The hyperfine values of the HCS and the isovalent $\mathrm{HCO}, \mathrm{HSiS}$ and $\mathrm{HSiO}$ radicals were calculated using the. B3LYP and MRSDCI methods by Chen and Huang [6]. Isotropic hyperfine coupling constants for the radicals $\mathrm{BH}_{2}, \mathrm{CH}_{2}^{-}, \mathrm{NH}_{2}, \mathrm{OH}_{2}^{+}$, $\mathrm{BH}_{3}{ }^{-}, \mathrm{CH}_{3}, \mathrm{NH}_{3}{ }^{+}, \mathrm{H}_{2} \mathrm{CO}^{+}$and $\mathrm{CH}_{2} \mathrm{CH}$ were obtained using the density-functional theory with a gradientcorrected local-spin-density approximation and a 
Slater-type-orbital basis set [7]. The hyperfine constants of aromatic radicals containing ${ }^{14} \mathrm{~N}$ nucleus were investigated by an extensive study in which there are 165 hyperfine constants belonging to 38 radical species [8]. They were obtained from the computations with the DFT/B3LYP and PBE0 functional combined with 6-31G*, N07D, TZVP, and EPRIII basis sets.

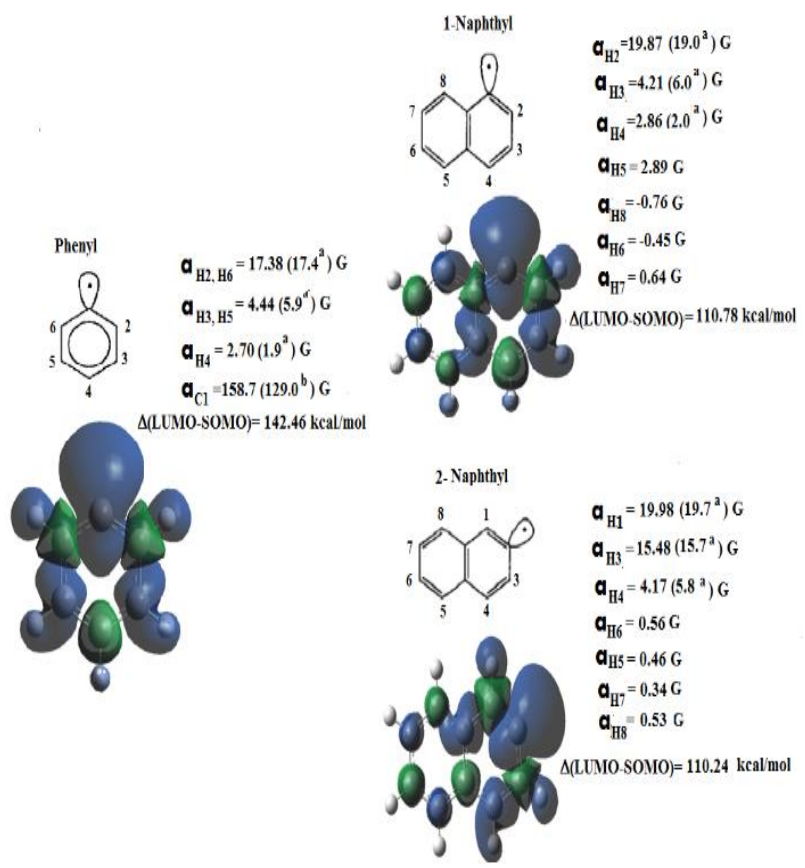

Figure 1. Calculated hyperfine constants and spin densities for phenyl and naphthyl radicals. ${ }^{a}$ Ref [4] and ${ }^{b}$ Ref. [13].

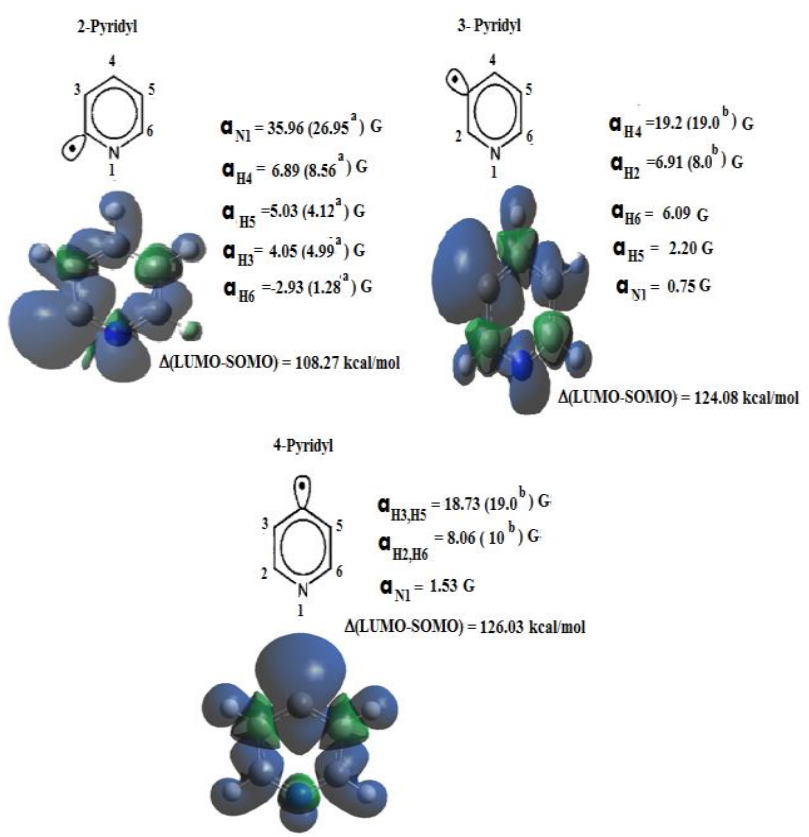

Figure 2. Calculated hyperfine constants and spin densities for pyridyl radicals. ${ }^{a}$ Ref [3] and ${ }^{b}$ Ref. [2].
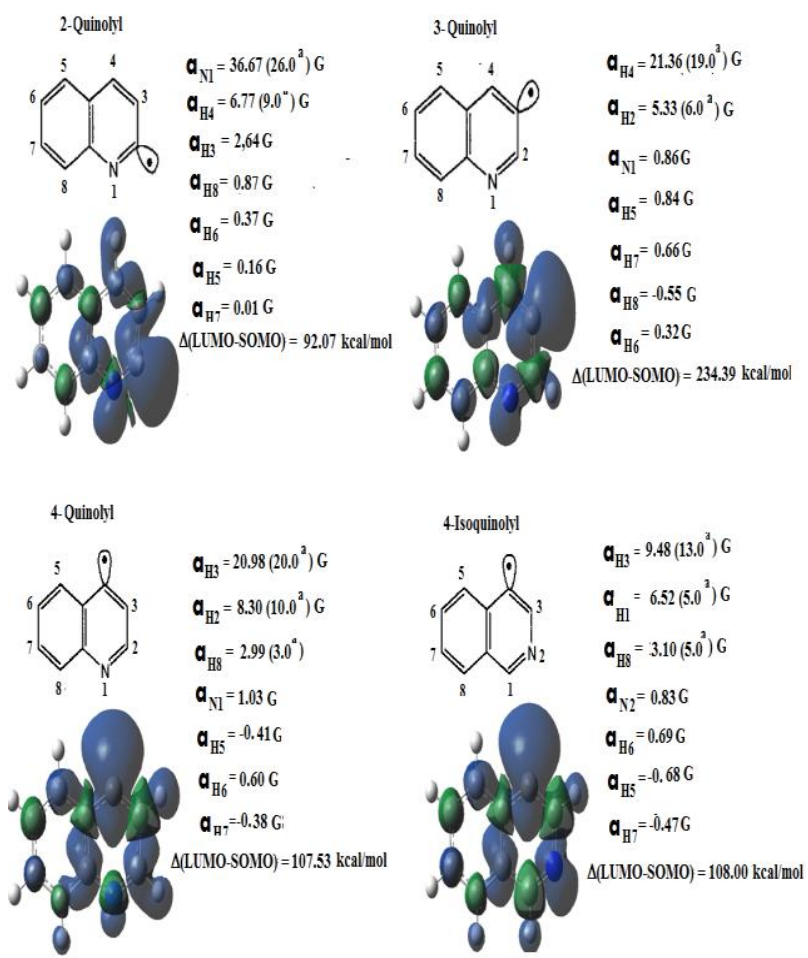

Figure 3. Calculated hyperfine constants and spin densities for quionolyl radicals. ${ }^{a}$ Ref [2].

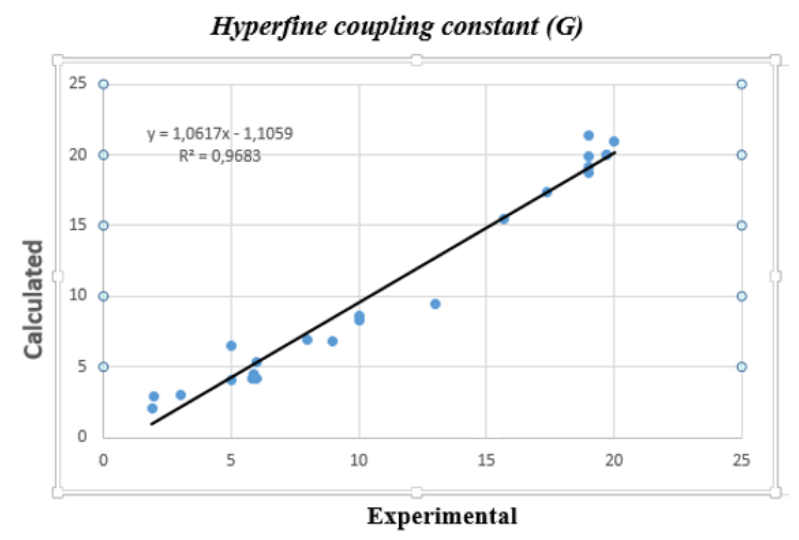

Figure 4. The correlation graph between the experimental and calculated hyperfine constants $(G)$.

In this study the isotropic hyperfine coupling constants of some $\sigma$-type radicals were calculated by DFT/B3LYP method at LAN2DZ level. It is aimed to determine the distributions of the spin density of these aromatic radicals.

\section{Computational Methods}

Our previous studies have showed that the DFT(B3LYP)/LanL2DZ level cause pretty good values in hyperfine calculations $[9,10]$. So in this study the radicals were optimized by using the DFT (B3LYP hybrid functional) method with LanL2DZ basis set level. All the calculations have been 
performed using Gaussian 09 package [11] and Gauss-View molecular visualization programs [12]. The simulated EPR spectra were obtained by using WINEPR SimFonia Version 1.25 software of Bruker.

\section{Results and Discussion}

The calculated hyperfine coupling constants (hfccs) and spin density distributions of all the radical have been shown in Figs. 1-3, respectively. Figures also show the experimental hfcc values. Taking into account that the calculated results based on a single gas molecule may not match to the experimental one in which multiple interactions takes place, there is reasonable agreement between the calculated and experimental values. Root-mean-square deviations (RMSD) between the experimental and computed hfccs of all the considered radicals except for two big values have been obtained and, given in Figure 4. From the RMSD value of about 1.97 in the figure, it is stated that the LanL2DZ basis set level gives a reasonable good agreement with the experimental data. The spin density distributions of all the radicals in Figs 1-3 show that all these radicals are $\sigma$-type radicals. The resolved EPR hyperfine structures of the radicals were readily identified with the hyperfine values of the hydrogen or nitrogen atoms of the radicals. From the figures we can say that the unpaired electron is localized mainly in one sphybrid orbital of the carbon atom from which hydrogen is removed and, that the spin densities on the ring hydrogen and nitrogen atoms are fairly low. The hffcs of only the close hydrogen or nitrogen atoms to the unpaired electron are enough big to visible in the EPR spectra of the radicals. The others are too low to appear in the linewidth of the EPR lines. They can only be calculated.

The highest occupied molecular orbital (HOMO) energy is associated with the electron donating ability of a molecule while the lowest unoccupied molecular orbital energy (LUMO) energy is an indicator of its electron accepting ability. HOMO is called as SOMO for semi occupied molecular orbital, namely radicals. The energy gap between $\mathrm{E}_{\text {номо }}$ and $\mathrm{E}_{\text {Lumo }}$ energy levels $(\Delta \mathrm{E})$ is an important parameter as a function of reactivity of a molecule. A molecule with a lower $\Delta \mathrm{E}$ is more chemical reactive. So, from the $\Delta \mathrm{E}$ values given in Figs.1-3, 2-naphthyl between naphthyl radicals, 2-pyridyl between pyridyl radicals and 2-quinolyl between quinolyl radicals has the highest chemical reactive.

Fig.5-7 shows the simulation and experimental EPR spectra of all the radicals. The simulations spectra were obtained by using the calculated hyperfine coupling constants of the radicals. The most corresponding linewith values are written in bottom of the figures. As seen the simulation and experimental spectra suit well. This shows the calculated values of hffc are in good agreement with the experimental data. The computations of the nonobserved hyperfine values completes the EPR analysis of all these radicals.
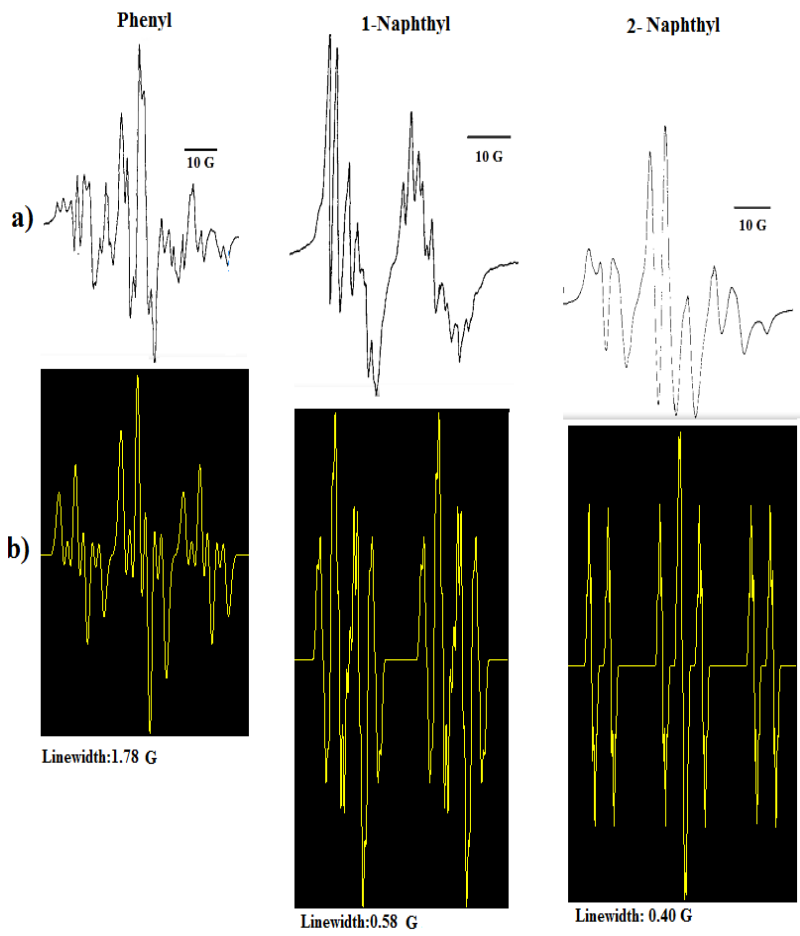

Figure 5. Simulation and experimental spectra of phenyl and naphthyl radicals. The experimental spectra was taken from Ref [4].

a)
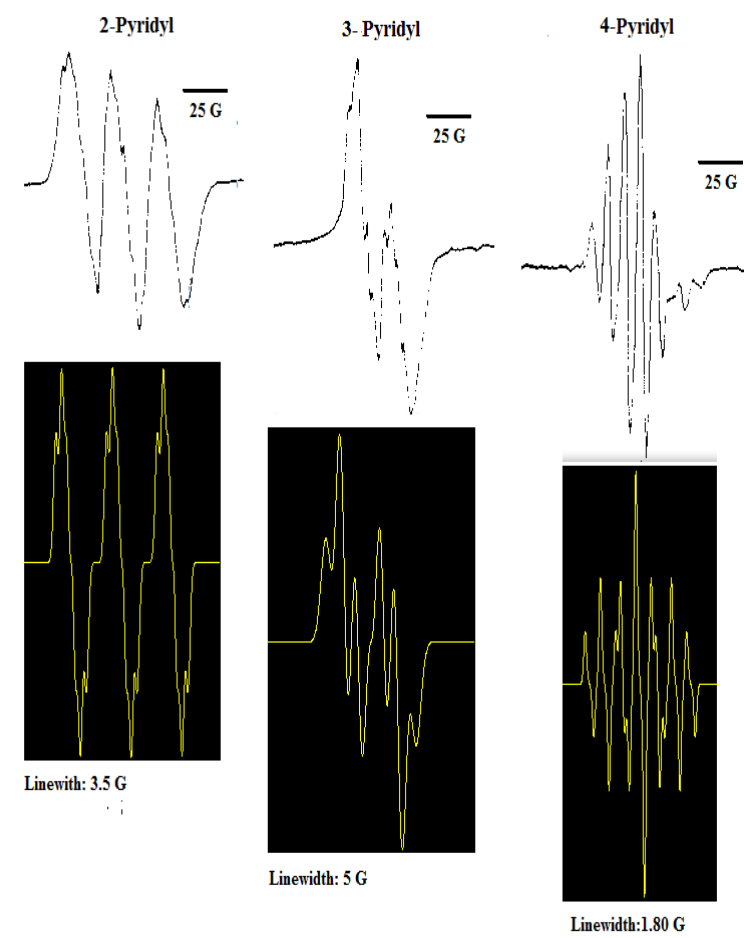

b)

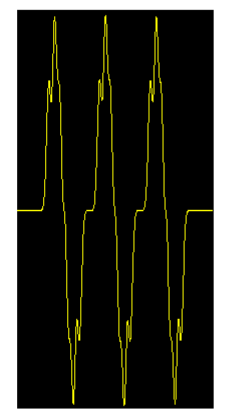

Linewith: $3.5 \mathrm{G}$

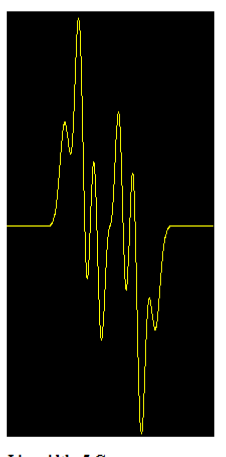

Figure 6. Simulation and experimental spectra of pyridyl radicals. The experimental spectra was taken from Ref [2]. 


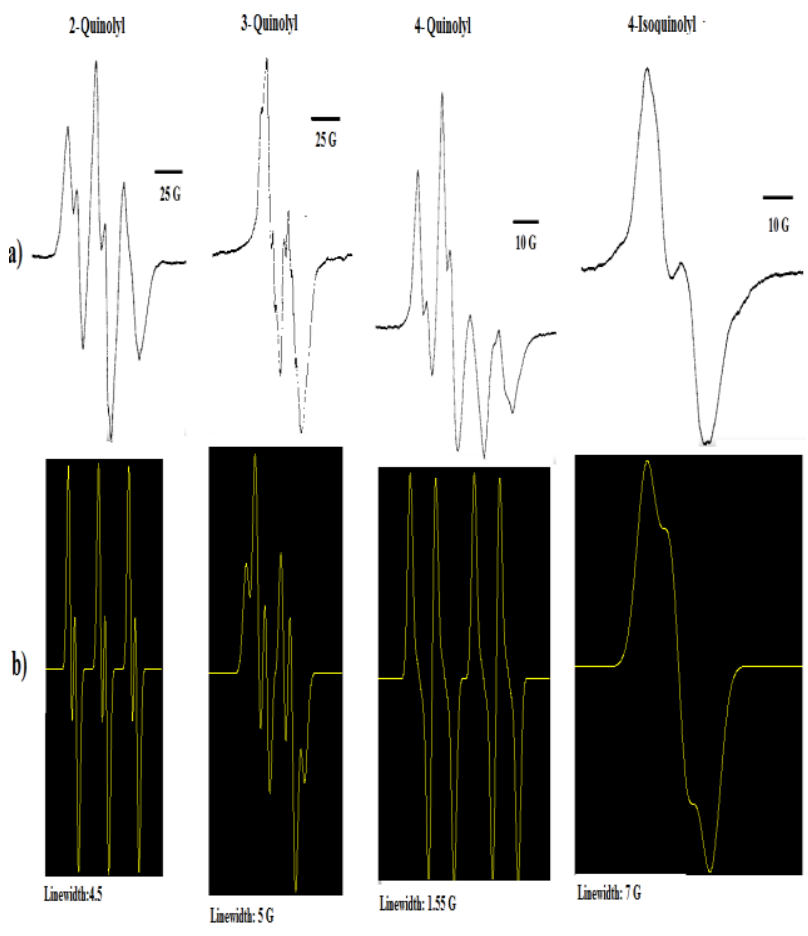

Figure 7. Simulation and experimental spectra of quoinolyl radicals. The experimental spectra was taken from Ref [2].

\section{Conclusions}

The EPR hyperfine coupling constants of some $\sigma-$ type phenyl, naphthyl, pridyl, and quinolyl radicals were calculated by using the DFT(B3LYP) method with LanL2DZ basic set. The simulated EPR spectra of all the radicals were also found by using the calculated isotropic hyperfine coupling constants and, compared with the experimental data. From the results it was concluded that the unpaired electron is localized mainly in one sp-hybrid orbital of the carbon atom from which hydrogen is removed, and that there is the effect of only the close hydrogen or nitrogen atoms of the fused benzene ring to the unpaired electron upon the EPR spectra of the radicals.

\section{References}

[1] P. H. Kasai and D. McLeod, "Electron spin resonance study of heterocycles. I. Pyridyl radicals" J. Americ. Chem. Soc. 94,3(1972)720-727. https://doi.org/10.1021/ja00758a007

[2] P. H. Kasai and D. McLeod, "Electron spin resonance study of heterocycles. V. Quinolyl radicals" J. Americ. Chem. Soc. 97,6 (1975) 1548-1551. https://doi.org/10.1021/ja00839a046
[3] H. Zemel and R. W. Fessenden, "Electron spin resonance studies of phenyl and pyridyl radicals in aqueous solution" J. Physic. Chem. 79,14 (1975) 1419-1427.

https://doi.org/10.1021/j100581a016

[4] P. H. Kasai, P. A. Clark, and E. B. Whipple, "Electronic ground states of aryl radicals" J. Americ. Chem. Soc. 92,9 (1970) 2640-2644. https://doi.org/10.1021/ja00712a009

[5] Bo-Z. Chen and Ming-B. Huang, "Hyperfine structure in HCS and related radicals: a theoretical" Chem. Phys. Lett. 308, 3-4 (1999) 256-262. https://doi.org/10.1016/S0009-2614(99)00601-6

[6] T. Wang, F. M. Tang and Y. F. Wu, “A combined EPR and DFT study of the overcrowded aromatic radical cations from Friedel-Crafts alkylation reactions" J. Mol. Struct. 1002,1-3 (2011). 128-134. https://doi.org/10.1016/j.molstruc.2011.07.009

[7] N. Ishii and T. Shimizu, "Density-functional-theory calculations of isotropic hyperfine coupling constants of radicals" Chem. Phys. Lett. 225,4-6 (1994). 462-466. https://doi.org/10.1016/0009-2614(94)87112-4

[8] L. Hermosilla, G. Vega, C. Sieiro, and P. Calle, "DFT Calculations of Isotropic Hyperfine Coupling Constants of Nitrogen Aromatic Radicals: The Challenge of Nitroxide Radicals" J. Chem. Theory Comput. 7, 1 (2011) 169-179. https://doi.org/10.1021/ct1006136

[9] S. Nardali, F. Ucun and M. Karakaya, "Calculated hyperfine coupling constants for 5,5-dimethyl-1pyrroline $\mathrm{N}$-oxide radical products in water and benzene" Russian J. Phys. Chem. A (2017) 91, (2017) 2137-2148. https://doi.org/10.1134/S0036024417110164

[10] F. Ucun and S.G. Aydın, "Calculated optimized structures and hyperfine coupling constants of some radical adducts of $\alpha$-phenyl- $N$-tert-buthyl nitrone in water and benzene solutions" J. Organomet. Chem. 759, (2014). 27-32. https://doi.org/10.1016/j.jorganchem.2014.02.011

[11] M. J. Frisch, G. W. Trucks, H. B. Schlegel, et al., Gaussian 09, Revision D.01 (Gaussian, Inc., Pittsburgh, PA, 2009). https://gaussian.com/g09citation/

[12] R. Dennington, T. Keith and J. Millam, GaussView, Version 5.0.9, (Semichem Inc., Shawnee Mission: KS, 2009). https://gaussian.com/g09citation/

[13] J. E Bennett and B. Mile "Electron Spin Resonance Spectra of Some -Type Aromatic Radicals" The J. Phys. Chem. 75, 22 (1971) 3423,3437. https://doi.org/10.1021/j100691a005 